\title{
Laban Movement Analysis towards Behavior Patterns
}

\author{
Luís Santos and Jorge Dias \\ Instituto de Sistemas e Robótica, \\ Departamento de Engenharia Electrotécnica e de Computadores \\ Faculdade de Ciência e Tecnologia da Universidade de Coimbra \\ Pólo II, Pinhal de Marrocos, Coimbra, Portugal \\ \{luis, jorge\}@isr.uc.pt
}

\begin{abstract}
This work presents a study about the use of Laban Movement Analysis (LMA) as a robust tool to describe human basic behavior patterns, to be applied in human-machine interaction. LMA is a language used to describe and annotate dancing movements and is divided in components [1]: Body, Space, Shape and Effort. Despite its general framework is widely used in physical and mental therapy [2], it has found little application in the engineering domain. Rett J. [3] proposed to implement LMA using Bayesian Networks. However LMA component models have not yet been fully implemented. A study on how to approach behavior using LMA is presented. Behavior is a complex feature and movement chain, but we believe that most basic behavior primitives can be discretized in simple features. Correctly identifying Laban parameters and the movements the authors feel that good patterns can be found within a specific set of basic behavior semantics.
\end{abstract}

Keywords: Laban Movement Analysis, Behavior Patterns, Bayesian Networks, Movement Characterization.

\section{Introduction}

Social interaction is a key issue in human life and also a relevant area in psychology and cognitive science. Social psychologists have been researching social interaction for a long time and conclude that social signals strongly determine human behavior. The majority of these signals are consciously produced, in the form of spoken language. However human interaction also involves non-verbal elements which are extensively and mainly unconsciously performed during human interaction. The nonverbal communication occurs concurrently to spoken words, through aural cues (voice quality, tone and fluency of speech) and visual cues (gestures, body language or posture, facial expression and gaze). These non-verbal signals are intuitively used by humans to understand and predict each other's behavior, mood, personality, and social relations, in a very wide range of situations. This work will focus on body's own language and movements.

By keeping track of relevant body parts (hands and head) a computational system can determine relevant features, thus providing ground for the estimation of parameters that will allow a robust analysis of performed actions and its characteristics [3]. 
The acquisition method is accomplished using video sensing devices. Image processing uses mainly two algorithms: CAMshift and Haar-like features to keep track of body parts. Once Cartesian data of body part position is available, basic Low Level Features (LLF) that constitute the basis of the Bayesian Network are computed. Detailed description of this acquisition and tracking methods is out of the scope of this work, and detailed information can be found in [3, 4, 5].

To interpret and contextualize the resulting LLF, Laban Movement Analysis (LMA) [1], a method for observing, describing, notating, and interpreting human movement, is used. Based on [3] we will debate how Computational Laban Movement Analysis (C-LMA) implemented over Bayesian Networks provides a good movement classifier. Bayesian Network properties allow the independent implementation of models, one for each of the Laban Components. By merging each of the models into one global model, it is believable that it will maximize result expectation for movement identification.

It has been shown that, in many social situations, humans can correctly interpret non-verbal signals and can estimate the behavior with high accuracy. Some approaches to characterize behavior decompose it in small basic actions performed in time. These actions exhibit certain characteristics, with some of them being personal i.e. specific for each individual. However it is felt necessary to create a system with an enough abstraction degree to characterize behavior in general. This work presents LMA as a potential tool to establish the relationship of mathematical properties of acquired visual cues to higher level semantic behavior description. This work aims to provide in the future a system that will automatically analyze behavior in different situations using the developed models based on Laban Movement analysis.

\section{Contribution to Technological Innovation}

Laban Movement Analysis is a tool widely used in studies of dance and in physical and mental therapy. However its computational implementation had yet been superficial. This work presents a computational approach to LMA implementation based on Bayesian Networks. The nature of this probabilistic method provides the necessary flexibility to model each of LMA components individually. Other interesting properties relates to the ability of Bayesian Framework to deal with incomplete information, uncertainty, make predictions on future events and, most important, provides an embedded scheme for learning. This work aims to take Rett's [3] work on movement classification using partial LMA implementation and extend the global model to a higher level: Behavior. LMA provides a set of descriptors carrying a semantic that can intuitively be seen as basic behavior characteristics. By developing LMA remaining components, hence completing the LMA global model, this work expects it will provide a good set of behavior descriptors based on LMA semantics. Behavior approaches so far, rely on pure geometric approaches, i.e. they do not carry inherent semantics which can immediately be related to behavioral aspects of human actions. Literature states that LMA provides good descriptors for the emotional content of expressive movements, thus, this work aims to take advantage of those characteristics and the flexibility given by the Bayesian approach to develop models to robustly characterize behavior. 


\section{State of the Art}

Laban Movement Analysis is a descriptive language to study dancing movements. The general framework is widely applied in physical and mental therapy [2] as well as studies on dance. However, it is finding little application in the engineering domain. Recently, researchers from neuroscience [6] started to investigate LMA as a tool to describe certain effects on the movements on animals and humans. [3] In his $\mathrm{PhD}$ Thesis within the Bayesian Approach to Cognitive Systems (BACS) project developed a social robot that could identify a set of movements within human-machine interaction. His robot interface interpreted movement using Bayesian models divided in abstraction levels based on Laban Movement Analysis.

Within behavior analysis are several studies based on probabilistic approaches. Despite the flexibility of the probabilistic approach, the majority of presented methods so far, lack the psychological aspects of behavior, focusing on purely geometrical approaches. Léon and Sucar [11] developed a Bayesian model to recognize different activities, with different speed of execution for different persons. They present a very small number of movements, characterized by the global trajectory of the movement between successive images. A few years later Hongeng et al. [8] presented a new method to recognize human activities. An activity is defined as being composed by events (simple or complex) executed by an individual, or through several events that model the interaction between individuals (multiple events). The event modeling, based on the body shape and trajectory of the subject is done through the hierarchical representation of activities Medioni [9], where events are organized in level of abstraction, allowing flexibility and modularity in activity modeling. Arsic [10] proposed a system to automatically detect abnormal behavior from passengers in public transport vehicles monitored by a fixed video camera. Environment constrains lead the authors to observe the passengers actions performed by the upper body. Arsic and their collaborators modeled behavior using Bayesian Networks, postulating that behaviors like nervous, aggressive and others could be described through the combination of a set of simple activities, performed by different body parts, which they called low level actions. Hence small actions like face expressions and global body movement (sitting, rising, etc.) constituted basic actions used by the system to identify high level behavior.

\section{Laban Movement Analysis}

Laban Movement Analysis (LMA) is a method to observe, describe, notate and interpret human movement, developed by Rudolf Laban (1879 to 1958). Foround et al. [6] states that LMA places emphasis on the underlying motor patterns by notating how the body segments are moving, how they are supported or affected by other body parts, as well as whole body movement. A recent study by Rett J. [3], explored how LMA can be used to classify human expressive movements within human-machine interaction. Rett's work also emphasizes that LMA, based in its inherent semantics, has the potential to analyze emotional content of human actions.

Laban theory consists of several major components, though the available literature does not set a standard regarding their total numbers. The work of Norman Badler's group [1, 7] mentions five major components: Body, Space, Effort, Shape and 
Relationship, though the latter is more abstract and deals/emerges from the other four, leading some studies to consider only the first four.

\subsection{Body}

The Body component deals with relative motion of body parts and the body center as well as which are moving at all. This component also deals with issues like locomotion and kinematics. More specifically, the kinematic chains try to relate the spatial Shaping and the inherent Effort qualities. Laban chose the navel as the body center. The lower part of the body is used to study the global locomotion and the upper body deals with exploring, manipulation and gesturing.

\subsection{Space}

The Space component presents concepts to describe the trajectory executed by the observed actor's body parts while performing a movement. These concepts are measured relative to a frame of reference determined by the body of the actor. Whilst concepts may differ in complexity of expressiveness and dimensionality, they are all reproducible in the 3-D Cartesian system and discretized in form of direction symbols [4]. Using observed trajectories, discretized in frames $I$ along time, Rett proposed the following Bayesian model $[3,4]$ to estimate the movement $M$ knowing 8 possible directions symbols $A$ in a $2-\mathrm{D}$ plane.

$$
\mathrm{P}(\mathrm{M} \mid \mathrm{A} I)
$$

This approach, whilst not presenting outstanding results (presented in section 5), was a good indicator regarding future developments. Hence Effort component was implemented.

\subsection{Effort}

What makes the framework of LMA so special is its ability to describe an additional 'expression' that accompanies the spatial trajectory (Space component). It relates lowlevel features like velocity, acceleration and curvature to Effort qualities like Time (E.Ti), Space (E.Sp), Weight (E.We) and Flow (E.Fl). By retrieving some evidences about the emotional state or the intention of the performer, the Effort component can be seen as the key descriptor to solve the task of analyzing 'expressive movements'. Table 1 shows the Effort qualities, the underlying cognitive process, the subject and the two extremes that characterize each quality has [2].

Table 1. Effort qualities and their subjects

\begin{tabular}{llll}
\hline Effort & Cognitive Process & Subject & Extremes \\
\hline Space & Attention & The Spatial Orientation & Direct / Indirect \\
Weight & Intention & The impact & Strong / Light \\
Time & Decision & The urgency & Sudden / Sustained \\
Flow & Progression & How to keep going & Free or Careful \\
\hline
\end{tabular}


Movements are described and distinguished by those qualities close to an extreme, e.g. a Punch movement has Strong Weight, Sudden Time and Direct Space. When a person acts sudden and vigorous/strongly, socially humans interpret these signs as someone who might be angry or violent, thus estimating themselves each other's behavior. It is reasonable to state that Effort descriptors approach the semantic humans use to characterize each other's movements in terms of "expressiveness"'. Following Rett's work, [12] extends and implements the Effort model (equation 2).

$$
P(M \mid \text { E.sp E.ti E.fl) }
$$

Knowing the Effort qualities Time E.ti, Space E.sp and Flow E.fl the movement $M$ can be estimated. Despite only Effort and Space have yet been implemented, one other component is now being studied that has special interest to the behavior concept is the Shape component. At this level of the Bayesian Network, movements are hand labeled with determined Effort characteristics, however in the lowest level, to decide which mathematical features are dominant in each of the Effort qualities, a probabilistic evaluation is made [3].

\subsection{Shape}

Bartenieff and Lewis [2] do not define Shape a component of its own but rather a set of qualities emerging from the Body and Space components. Two Shape qualities were mentioned particularly: Shape Flow describes movements that are focused on the body itself, going towards or away from the body center and using descriptors like shrinking and growing, bulging and hollowing (also including breathing). The term Spatial Shaping is used for movements that are going towards a goal in space (e.g. reaching). It is usually described in a Euclidean frame of reference that is aligned with an initial position of the egocentric frame of reference. Due to this, movements can be described by using the vertical, horizontal and sagittal axes and relating them to bipolar descriptors like sinking and rising, enclosing and spreading, and retreating and advancing. Fig 1. shows the descriptors (left) and some exemplary movements (right).
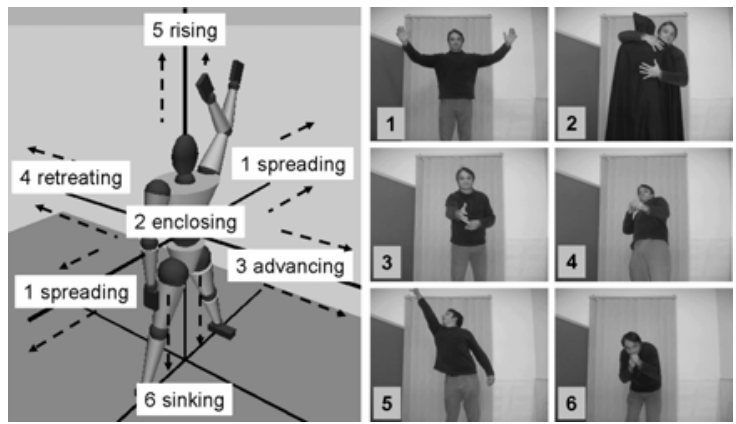

Fig. 1. The Shape component with its spatial qualities and some exemplary movements: 1 . embracing, 2. hugging, 3. shake, 4. retreating 5.reaching and 6.ducking. 


\section{Practical Implementation}

In the referred studies [4], to test the implemented Space model, the actor performed the movements facing one vision acquisition system. The available database encompassed eight previously learned movements, yielding this model to present 32 misclassification trials out of 95 , yielding a positive classification rate of $66,31 \%$.

To study Effort model [12] a database of 5 movements was designed. These movements were spatially similar, however characterized for having different combinations of Effort qualities. Table 2 summarizes the obtained results.

Table 2. Results for Effort qualities

\begin{tabular}{lcccccc}
\hline & \multicolumn{6}{c}{ Physical qualities } \\
\cline { 2 - 7 } & \multicolumn{2}{c}{ Space } & \multicolumn{2}{c}{ Flow } & \multicolumn{2}{c}{ Time } \\
\cline { 2 - 7 } & Ind & Dir & Free & Bound & Sud & Sus \\
\hline $\begin{array}{l}\text { Positive } \\
\text { Results }\end{array}$ & $79.3 \%$ & $90.2 \%$ & $61.2 \%$ & $58.7 \%$ & $84.8 \%$ & $97.1 \%$ \\
\hline
\end{tabular}

In light of the presented results, LMA starts to present its true potential. It was demonstrated that spatial similar movements can be discretized using Effort parameters. The potential becomes clearer when the two already implemented models are fused in one global model (equation 3). Results are presented in Table 3.

$$
P(M \mid A \text { I E.sp E.ti E.fl })
$$

Table 3. Results for Effort and Space combined

\begin{tabular}{lccc}
\hline & \multicolumn{3}{c}{ Laban Components } \\
\cline { 2 - 4 } & Space & Effort & Space + Effort \\
\hline Classification Rate & $61.3 \%$ & $86,4 \%$ & $79.4 \%$ \\
\hline
\end{tabular}

An improvement of the overall classification can be seen. One can argue that Effort alone provides better results than the joint global model. However, it must be taken into consideration that the studies on Effort model alone used a specific database for proof of concept. The experiments with the global model took both databases used in Effort and Space. By implementing the two remaining components we expect to strengthen the global model, leading to a good movement/body language characterization in terms of Laban descriptors.

A robust tracking system has been developed to deal with actors pose changes. It is based on stereo camera system which allows the system to track body parts in 3-D space. The methodology is out of the scope of this work. Details can be found in [5]. 


\section{Behavior Modeling}

The previous section provided and debated LMA modeling results based on Bayesian network, demonstrating that the used probabilistic approach could potentiate a computational implementation of a this valuable tool for human movement characterization. Behavior has been studied $[8,10,11]$ and the common fact these approaches have, is that behavior can be composed of small actions/events. With this concept in mind, this work proposed an approach based on Laban Movement Analysis, where behavior is divided in small movements and Laban parameters. Actions performed by human beings carry themselves emotion that can be expressed in form of visual cues: movements and body language. Rett [3] demonstrated that LMA could be used to identify movements. Literature $[1,2]$ states, that Laban semantics present a natural language to express body motion. Descriptors like sudden, strong, sinking or stretching can very well be applied when humans are trying to understand each other's behavior. Hence by knowing each of these characteristics, one can infer basic behavior actions. Using a Bayesian language, knowing movement $M$ and Laban components Comp the behavior $B$ can be estimated.

$$
P(B \mid M \text { Comp })
$$

Equation (5) presents the possible space for the variable $B$

$$
B \in\{\text { Violent, Relaxed, Quiet, (...)\} }
$$

Equation (4) is the first approach to a behavior Bayesian model, and evolution of the presented work makes it a believable and innovative solution for an expected robust behavior probabilistic characterization.

\section{Conclusions}

The implemented LMA components presented positive results. As results demonstrated, the merging of two components improved classification, which leads to the expectation of further classification improvement with the implementation of the other Laban components in future work. Also Laban parameters are found to be good behavior descriptors with an enough abstraction degree to give models the necessary flexibility. Behavior can be divided in small events, and described through Laban parameters. Future work will lead to the final LMA global implementation and behavior parameterization with the aim to provide a robust behavior characterizer to be applied in areas like rehabilitation or surveillance.

\section{References}

1. Zhao, L.: Synthesis and Acquisition of Laban Movement Analysis Qualitative Parameters for Communicative Gestures. PhD Thesis, University of Pennsylvania (2002)

2. Bartenieff, I., Lewis, D.: Body Movement: Coping with the Environment. Gordon and Breach Science, New York (1980) 
3. Rett, J.: Robot Human Interface Using Laban Movement Analysis Inside a Bayesian Framework. PhD Thesis, University of Coimbra (2009)

4. Rett, J., Santos, L., Dias, J.: Laban Movement Analysis using Multi-Ocular System. In: International Conference on Intelligent Robots and Systems, IROS (2008)

5. Prado, J., Santos, L., Dias, J.: Horopter based Dynamic Background Segmentation applied to an Interactive Mobile Robot. In: 14th International Conference on Advanced Robotics, ICAR (2009)

6. Foroud, A., Whishaw, I.Q.: Changes in the kinematic structure and non-kinematic features of movements during skilled reaching after stroke: A Laban movement analysis in two case studies. Journal of Neuroscience Methods 158, 137-149 (2006)

7. Chi, D., Costa, M., Zhao, L., Badler, N.: The emote model for effort and shape. In: SIGGRAPH 2000, Computer Graphics Proceedings. Annual Conference Series, ACM SIGGRAPH, pp. 173-182. ACM Press, New York (2000)

8. Hongeng, S., Nevatia, R., Bremond, F.: Video-based event recognition: activity representation and probabilistic recognition methods. Computer Vision and Image Understanding 96, 129-162 (2004)

9. Medioni, G., Cohen, I., Bremond, F., Hongeng, S., Nevatia, R.: Event detection and analysis from video streams. IEEE Transactions on Pattern Analysis and Machine Intelligence 23, 873-889 (2001)

10. Arsic, D., Wallhoff, F., Schuller, B., Rigoll, G.: Video based online behavior detection using probabilistic multi-stream fusion. In: Proceedings of the IEEE International Conference on Image Processing, vol. 2, pp. 606-609 (2005)

11. León, R.D., Sucar, L.E.: Continuous activity recognition with missing data. In: Proceedings of the 16th International Conference on Pattern Recognition, vol. 1, pp. 439-442 (2002)

12. Santos, L., Prado, J., Dias, J.: Human Robot Interaction Studies on Laban Human Movement Analysis and Dynamic Background Segmentation. In: 2009 IEEE/RSJ International Conference on Intelligent Robots and Systems, IROS (2009) 